\title{
Terminal-selective functionalization of alkyl chains by regioconvergent cross-coupling
}

\author{
Stéphanie Dupuy, Ke-Feng Zhang, Anne-Sophie Goutierre, and Olivier Baudoin*
}

\begin{abstract}
To date, hydrocarbons are still the most important precursors of functionalized organic molecules, which has stirred the discovery of new $\mathrm{C}-\mathrm{H}$ bond functionalization methods. We describe herein a new step-economical approach, which allows to build new $C-C$ bonds at the terminal position of linear alkanes. First, we show that secondary alkyl bromides can undergo in situ conversion to alkylzinc bromides and regioconvergent Negishi coupling with aryl or alkenyl triflates. The use of a suitable phosphine ligand favoring Pd migration allows to obtain the major linear cross-coupling product. Subsequently, mixtures of secondary alkyl bromides were prepared from linear alkanes by standard bromination, which upon regioconvergent cross-coupling allowed to access linear arylated product in only two steps.
\end{abstract}

The direct functionalization of hydrocarbon chains represents a stepeconomical approach to tetrahedral carbon-rich functionalized organic intermediates from cheap and abundant feedstock, and has recently been a topic of intense investigations. ${ }^{[1]}$ Two main strategies employing transition-metal catalysts have been pursued to tackle this challenging synthetic problem: ${ }^{[2]}$ inner-sphere $\mathrm{C}-\mathrm{H}$ activation, involving concerted $\mathrm{C}-\mathrm{H}$ bond cleavage and carbon-metal bond formation, and outer-sphere $\mathrm{C}-\mathrm{H}$ activation, not involving organometallic intermediates, which show distinct selectivity profiles. In most reported inner-sphere reactions, a directing group is employed to control the site-selectivity of the $\mathrm{C}-\mathrm{H}$ bond cleavage, whereas non-directed methods are scarce. ${ }^{[1 \mathrm{~b}]}$ The transition metal-catalyzed $\quad \mathrm{C}-\mathrm{H}$ borylation reactions developed by Hartwig and co-workers constitute notable examples, wherein terminal-selective activation and functionalization of alkyl chains is achieved by steric control (Scheme 1a). ${ }^{[3,4]}$ This borylation reaction can be followed by a Pd-catalyzed Suzuki-Miyaura chains. cross-coupling in order to create a $\mathrm{C}-\mathrm{C}$ bond at the same position (2 $\rightarrow 3$ ), with the inconvenient that two noble metals are employed in this two-step sequence. ${ }^{[5]}$ A conceptually different strategy is reported herein (Scheme 1b). Secondary bromoalkanes $\mathbf{4}$ are readily available compounds, which can be generated by bromination of abundant feedstock such as alkenes, alcohols, or even alkanes $\mathbf{1}$. The generation of an alkylzinc halide 5 from $\mathbf{4}$ by metal insertion, ${ }^{[6]}$ followed by transmetallation to an in situ-generated organopalladium complex $\left[\mathrm{Pd}\left(\mathrm{R}^{2}\right) \mathrm{YL}\right]$ would form the corresponding branched alkylpalladium intermediate $\mathbf{6}$. The latter would migrate to the terminal position $(\rightarrow 7)$ via a succession of $\beta$ $\mathrm{H}$ elimination, rotation and insertion steps induced by a suitable ancillary ligand, where it would undergo reductive elimination to give the linear product 3. ${ }^{[7,8]}$ Ligand-controlled $\mathrm{Pd}^{0}$-catalyzed migrative couplings have been investigated by our group using enolates as nucleophiles ${ }^{[9]}$ or organozinc compounds obtained by Boc-directed lithiation and Li-Zn transmetallation. ${ }^{[10]}$ In the current work, we show that terminal (linear) cross-coupling products 3 can be obtained in a regioconvergent manner in just one step from

a) example of terminal-selective functionalization of alkyl chains

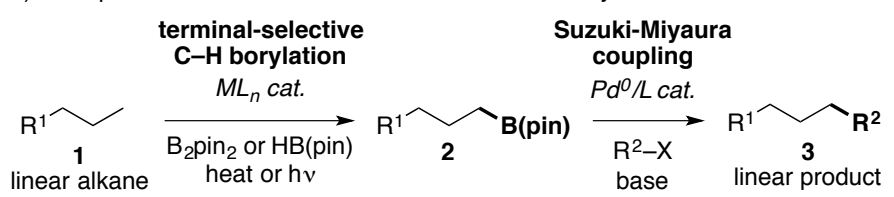

b) this work: a new terminal-selective functionalization strategy based on ligand-controlled Pd migration

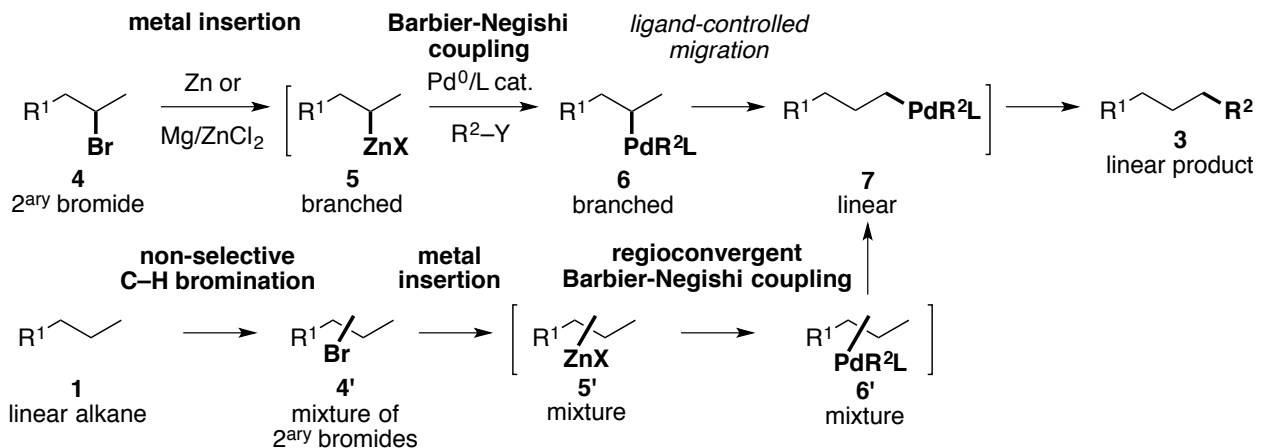

Scheme 1. Two conceptually different strategies for the terminal-selective functionalization of alkyl

bromoalkanes 4 under operationally simple, Barbier (in situ) conditions. In addition, in most cases a similar selectivity pattern is obtained regardless of the position of the bromine atom in the alkyl chain. This observation led us to employ mixtures of bromides $4^{\prime}$ obtained by radical bromination of linear alkanes $\mathbf{1}$. Indeed, this process is known to give rise to mixtures of secondary bromides, ${ }^{[11]}$ and hence has been of little synthetic use so far. However, we reasoned that this mixture could be productively utilized by applying the same in situ metal insertion/migrative Negishi coupling sequence, wherein a mixture of branched organopalladium intermediates $\mathbf{6}^{\prime}$ ' would converge to the same linear complex $\mathbf{7}$, 
thereby furnishing the same linear product 3 .

At the outset of our studies, we sought suitable and practical conditions to generate $(\mathbf{4} \rightarrow \mathbf{5})$ and cross-couple $(\mathbf{5} \rightarrow \mathbf{3})$ alkylzinc reagents in situ, in order to maximize the overall step economy. Such direct Barbier-Negishi couplings have been reported by Lipshutz and co-workers, who employed water as solvent and a diamine as additive, either in the presence of a surfactant ${ }^{[12]}$ or under "on-water" conditions. ${ }^{[13]}$ An improved catalytic system, allowing to couple a range of cyclic bromoalkanes and aryl electrophiles, has been recently disclosed by Buchwald and co-workers. ${ }^{[14]}$ However, to the best of our knowledge, alkyl-aryl couplings have not been reported using non-aqueous conditions. ${ }^{[15,16]}$ We reasoned that aryl triflates (Y = OTf) would be suitable electrophiles, since they should not undergo zinc insertion, and therefore should be orthogonal to alkyl halides 4. Hence, we started to investigate the coupling of aryl triflate 8a with an excess of the organozinc reagent generated from 2-bromopropane $\mathbf{4 a}$, zinc dust and $\mathrm{LiCl}$ in $\mathrm{THF},{ }^{[6 \mathrm{a}]}$ in the presence of a Pd catalyst formed in situ from $\mathrm{Pd}_{2} \mathrm{dba}_{3}$ and a suitable phosphine ligand (Table 1). The choice of the latter was guided by our previous studies on migrative Negishi couplings, wherein flexible phenylazole-based ligands were found to induce the highest selectivities in favor of the linear product. ${ }^{[10]}$ After testing a library of home-made ligands from this family (Table S1), we found that phenyl-pyrrole-based phosphine $\mathbf{L}^{1}$ containing $n$-butyl $P$ substituents, which is easily synthesized in one step from $N$ - phenylpyrrole, provided the highest linear selectivity, presumably due to its high degree of flexibility at both the phenyl-pyrrole and the P-alkyl bonds. Gratifyingly, using $5 \mathrm{~mol} \% \mathrm{Pd}$ and $7.5 \mathrm{~mol} \%$ of $\mathbf{L}^{1}$, a mixture of linear (3a) and branched (9a) products was obtained with good selectivity $(9: 1)$ albeit very low yield, presumably caused by the slow zinc insertion (entry 1). We thus looked for conditions allowing to generate the organozinc intermediate more rapidly. Gratifyingly, under Knochel's conditions employing a mixture of $\mathrm{Mg}, \mathrm{LiCl}$ and $\mathrm{ZnCl}_{2},{ }^{[6 b]}$ the yield was greatly improved, whereas a good linear/branched (l/b) selectivity was maintained (entry 2). Interestingly, omitting zinc chloride afforded similar results, starting either from 2-bromopropane 4a or 2-chloropropane $4 \mathbf{b}$ (entries 3-4). A control experiment with the commercially available, pre-formed Grignard reagent $\mathbf{4 c}$ also led to a similar outcome (entry 5). Of note, 2-chloropropane also proved a competent reactant in the presence of $\mathrm{ZnCl}_{2}$, albeit with a lower yield (entry 6). Importantly, when the more rigid and bulky phosphine $\mathbf{L}^{2}$ developed by Buchwald and coworkers was employed, ${ }^{[17]}$ the selectivity was completely reversed, thus leading exclusively to the branched product 9a in $82 \%$ yield (entry 7). These results highlight the role of the ligand in the linear/branched selectivity control. Moreover, they show that both linear and branched products can be synthesized in a divergent manner from the same reactants by choosing a different ligand. The reaction of aryl triflate $\mathbf{8 b}$ containing the more electrophilic ester substituent was next studied to further probe the utility of the $\mathrm{Mg}$ to $\mathrm{Zn}$ transmetallation (entries 8-9). As suspected, adding $\mathrm{ZnCl}_{2}$ proved to be crucial to perform the desired coupling successfully (entry 9), since only low amounts of coupling product were observed in its absence (entry 8 ). These results show that $\mathrm{ZnCl}_{2}$ can be omitted for less sensitive substrates such as 8a (entries 3-5), but also that the $\mathrm{Mg} / \mathrm{LiCl} / \mathrm{ZnCl}_{2}$ system should provide better chemoselectivity and versatility. Final refinements of reaction conditions were achieved by diminishing the quantity of in situ-generated organozinc reagent to 2 equiv (entry 10), and by lowering the catalyst loading to $2.5 \mathrm{~mol} \% \mathrm{Pd}$ and ligand (entry 11). Further decreasing the loading to $1.25 \mathrm{~mol} \% \mathrm{Pd}$ turned out to be viable, but the yield was lower (entry 12). Finally, the reactions of $\mathbf{8 a}$ with deuterated 1- and 2-bromopropane showed a pronounced deuterium scrambling over the three carbons, thereby indicating that palladium migrates in a reversible fashion along the alkyl chain (Figure S1).

The substrate scope was next studied under the optimal conditions employing $2.5 \mathrm{~mol} \%$ of Pd catalyst (Scheme 2). The catalyst loading was doubled when incomplete conversions were observed. We first investigated the reactivity and selectivity of structurally diverse linear alkyl bromides, that were either commercially available, or synthesized in a few steps via standard chemistry, using triflate $\mathbf{8 a}$ as an electronically and sterically unbiased electrophile (Scheme 2a). All three isomers of bromopentane $\mathbf{4 d - f}$ gave rise to the same coupling product $\mathbf{3 c}$ with $1 / \mathrm{b}$ selectivities above 90:10 in the presence of ligand $\mathbf{L}^{1}$. In contrast, replacing $\mathbf{L}^{1}$ with $\mathrm{CPhos} \mathbf{L}^{\mathbf{2}}$ led to a mixture of both branched products, and no linear product was observed. The more sensitive bromoesters $\mathbf{4 g}$-h furnished product $\mathbf{3 d}$ with a slightly reduced selectivity (87:13-89:11). However, these examples further demonstrate the good chemoselectivity of this process. Surprisingly, isomeric bromides $\mathbf{4 i - j}$ containing a phenyl ring led to the major linear product $\mathbf{3 e}$ with significantly different selectivities. Moreover, the reaction of bromide $\mathbf{4 k}$ did not give any coupling product. These results 


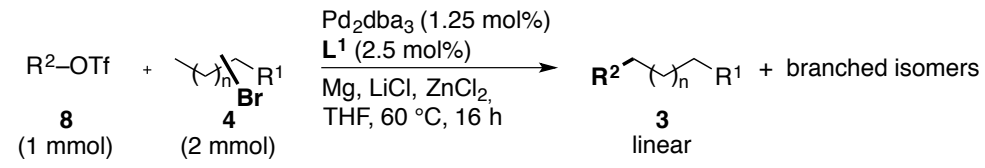

a) alkyl bromides ( $\left.\mathrm{R}^{2}-\mathrm{OTf}: \mathbf{8 a}\right)$

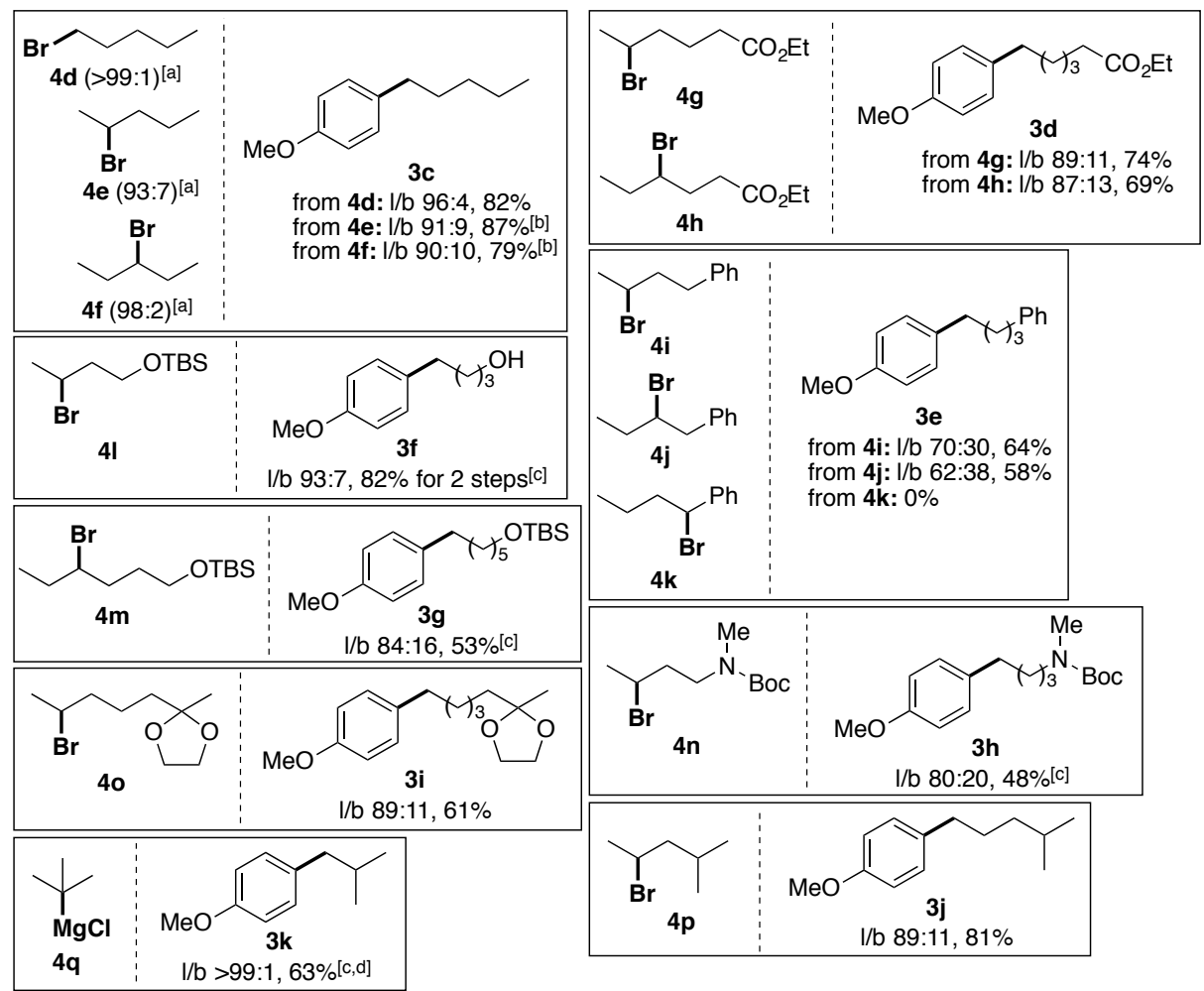

b) aryl/alkenyl triflates (alkyl bromide: $\mathbf{4 f}$ )

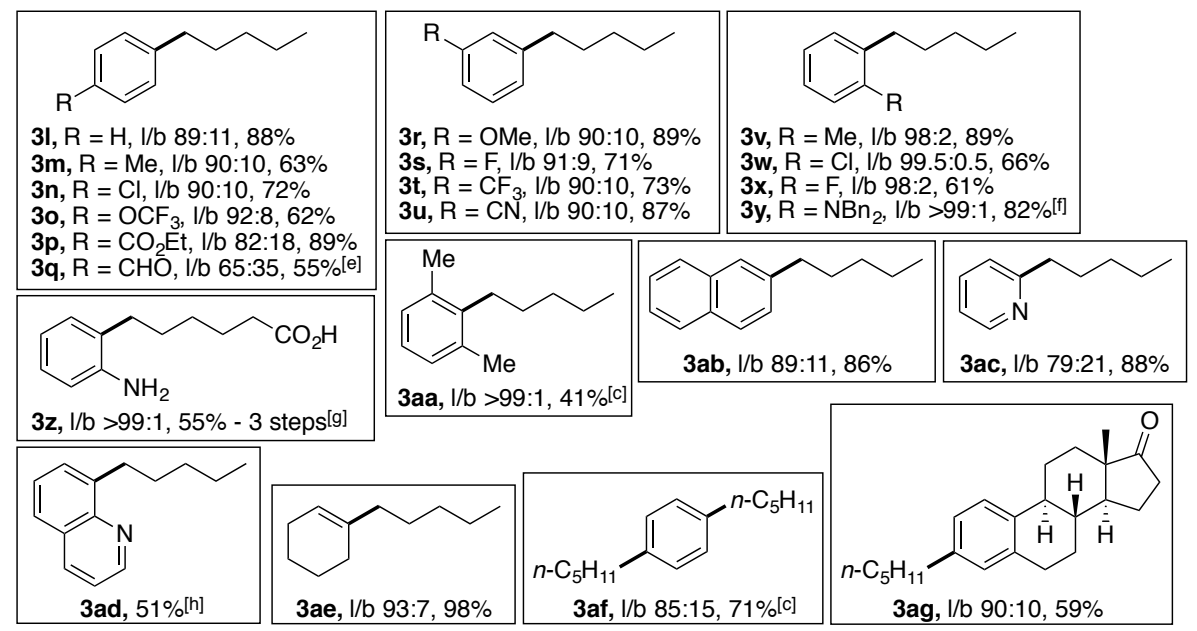

Scheme 2. Scope and limitations of the regioconvergent cross-coupling. Standard reaction conditions: $\mathrm{R}^{2} \mathrm{OTf} 8$ (1 mmol), alkyl bromide 4 (2 equiv), $\mathrm{Mg}$ (2 equiv), $\mathrm{LiCl}$ (2 equiv), $\mathrm{ZnCl}_{2}$ (2 equiv), $\mathrm{Pd}_{2} \mathrm{dba}_{3}(1.25 \mathrm{~mol} \%), \mathrm{L}^{1}(2.5 \mathrm{~mol} \%), \mathrm{THF}, 60{ }^{\circ} \mathrm{C}, 16 \mathrm{~h}$. Linear/branched ratios were measured by GCMS. Yields refer to the isolated linear/branched mixture. [a] Isomeric purity of starting bromides determined by GCMS; for $\mathbf{4 e}$ and $\mathbf{4 f}$, the minor isomer was the other secondary bromide. [b] With $\mathbf{L}^{2}$ as the ligand, a mixture of 2- and 3-arylpentanes was obtained with ratios of 93:7 for 4 e and 70:30 for $\mathbf{4 f}$ with no trace of linear product 3c. [c] Using $2.5 \mathrm{~mol}^{2} \mathrm{Pd}_{2} \mathrm{dba}_{3} / 5 \mathrm{~mol} \% \mathrm{~L}^{1}$. [d] With $\mathrm{L}^{2}$ as the ligand, a $2: 1$ ratio of $3 \mathrm{k}$ /anisole was obtained. [e] Using 4 equiv $\mathrm{ZnCl}_{2}$. [f] With $\mathrm{L}^{2}$ as the ligand, the I/b ratio was 73:27. [g] i) migrative cross-coupling; ii) $\mathrm{H}_{2}, \mathrm{Pd} / \mathrm{C}$, EtOH (55\% for 2 steps); iii) $\mathrm{LiOH}$, $\mathrm{THF} / \mathrm{H}_{2} \mathrm{O}$ (quant.). [h] Yield of the pure linear product; in this case the $\mathrm{l} / \mathrm{b}$ ratio could not be determined.
In addition to an ethyl ester (3d), the reaction was found to be compatible with other useful functional groups such as a TBSprotected alcohol (4l-m), a Bocprotected secondary amine (3h), and an acetal (3i). In particular, TBS-protected bromide $4 \mathbf{l}$ led to alcohol $\mathbf{3 f}$ in both excellent yield (82\% for two steps after acidmediated deprotection of the TBS group), and selectivity (1/b 93:7). The migrative arylation of the longer-chain TBS-protected bromoalcohol $\mathbf{4 m}$, wherein the bromine atom was installed at a more central position of the alkyl chain (similar to $\mathbf{4 h}$ ), also proceeded with acceptable yield and good selectivity. Interestingly, the reaction of 2-bromo-4methylpentane $\mathbf{4 p}$ containing a tertiary carbon led to compound $\mathbf{3 j}$ as the sole linear product with good yield and $89 \%$ selectivity. No trace of the other possible linear product was observed, thereby indicating that branching arrests $\mathrm{Pd}$ migration along the alkyl chain. Tertiary halides such as tert-butyl bromide and iodide failed to react under the same Barbier-type conditions. We hypothesized that this lack of reactivity might originate from the $\mathrm{Mg}$ insertion step, which is known to be very slow with tertiary halides. ${ }^{[18]}$ Hence, we directly engaged commercially available tert-butylmagnesium chloride $\mathbf{4 q}$, which indeed gave rise to the corresponding linear product $\mathbf{3 k}$ in $63 \%$ yield with no trace of branched product. The same selectivity in favor of the linear product was obtained with ligand $\mathbf{L}^{2}$ and it is therefore essentially substratecontrolled with this substrate, but in this case product $\mathbf{3 k}$ was accompanied by large amounts of anisole and could not be isolated from the crude mixture - therefore ligand $\mathbf{L}^{\mathbf{1}}$ seems to be the most suitable ligand for tertiary substrates as well.

Next, the scope with regard to the triflate was investigated using 3bromopentane $4 \mathbf{f}$ as the alkyl bromide (Scheme 2b). The reaction conditions were found to be compatible with a variety of electron-poor and electron-rich 
substituents on the para (3l-p) and meta (3r-u) positions, thereby providing the linear product with good yields $(62-89 \%)$ and $1 / b$ selectivities (82:18-92:8). A chlorine atom (3n) and electrophilic ester and nitrile functional groups (3p, 3u) were well tolerated. Even the more electrophilic aldehyde $\mathbf{3 q}$ could be obtained, albeit in lower yield and selectivity despite the use of a larger excess of $\mathrm{ZnCl}_{2}$ to prevent the nucleophilic addition of the Grignard intermediate. Ortho substituents provided higher 1/b ratios $(3 \mathbf{v}-\mathbf{y})$, consistent with previous work. ${ }^{[9,10]}$ A control experiment with ligand $\mathbf{L}^{2}$ showed that the selectivity is both substrate and ligand-controlled in this case (for product $3 \mathbf{y}$ : 1/b $>99: 1$ with $\mathbf{L}^{1}$ and 73:27 with $\mathbf{L}^{2}$ ). An interesting combination of alkyl bromide and aryl triflate featuring bromoester $\mathbf{4 g}$ and ortho-dibenzylaminophenyl triflate allowed to synthesize original $\eta$-aminoacid $\mathbf{3 z}$ in $55 \%$ overall yield for three steps after hydrogenation and ester hydrolysis. Other interesting aryl triflates included those containing two ortho substituents (3aa), a naphthyl ring (3ab), and a heteroarene (3ac-ad). Interestingly, as shown with product 3ae, the reaction is not limited to (hetero)aryl triflates, and cyclohexenyl triflate also gave excellent results (1/b 93:7, 98\%). In addition, a bis-triflate reacted on both sides, to give the double cross-coupling product 3af in good yield and selectivity. Finally, a more complex estrone-derived triflate was reacted successfully (3ag).

As a proof-of-concept experiment toward the development of a two-step linear-selective functionalization of alkanes, an equimolar mixture of bromopentanes 4d-f was prepared and reacted with aryl triflate 8a under the standard cross-coupling conditions (Scheme 3a).

a) mixture of bromopentanes

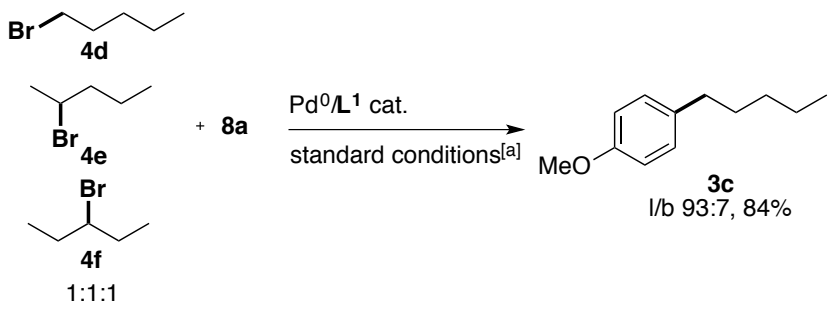

b) two-step $\mathrm{C}-\mathrm{H}$ functionalization sequence from alkanes

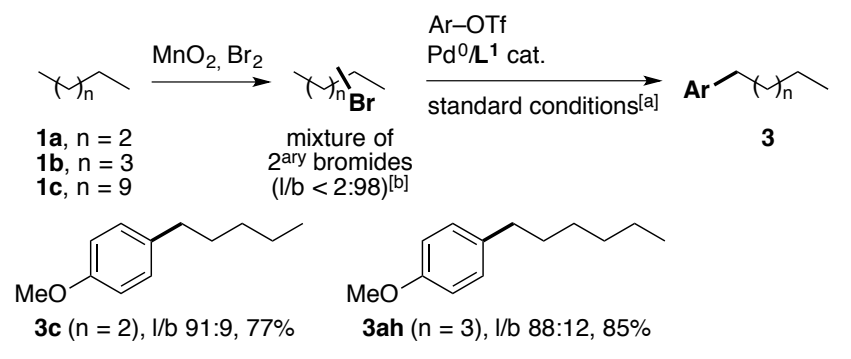

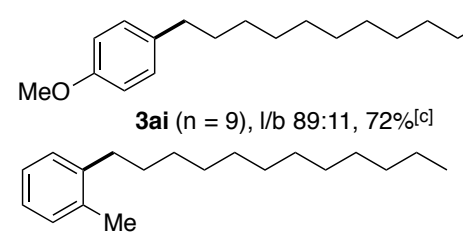

3aj $(n=9), 1 / b$ 97:3, 65\%[c]

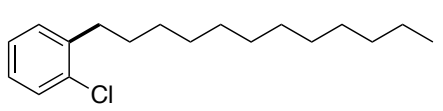

3ak $(n=9), 1 / b>99: 1,67 \%$ $61 \%(1.7 \mathrm{~g})$ on $10 \mathrm{mmol}$ scale
Scheme 3. Development of a two-step functionalization of linear alkanes. Linear/branched ratios were measured by GCMS. Yields refer to the isolated linear/branched mixture. [a] ArOTf $(1 \mathrm{mmol})$, mixture of alkyl bromides (2 equiv), $\mathrm{Mg}$ (2 equiv), $\mathrm{LiCl}$ (2 equiv), $\mathrm{ZnCl}_{2}$ (2 equiv), $\mathrm{Pd}_{2} \mathrm{dba}_{3}$ (1.25 mol\%), L ${ }^{1}$ (2.5 mol\%), THF, $60{ }^{\circ} \mathrm{C}, 16 \mathrm{~h}$. [b] Isomeric composition for pentane: 2-bromopentane/3-bromopentane 79:21; for hexane: 2 bromohexane/3-bromohexane 59:41; for dodecane: mixture of five secondary bromododecane isomers 19:17:20:18:26. [c] Using $2.5 \mathrm{~mol} \%$ $\mathrm{Pd}_{2} \mathrm{dba}_{3} / 5 \mathrm{~mol} \%$ of $\mathrm{L}^{1}$.
The corresponding linear product 3c was obtained with a yield (84\%) and a selectivity (1/b 93:7) which are consistent with the results obtained with isolated bromopentanes (see Scheme 2a, top left). This result suggested that the same principle would be applicable to perform the regioconvergent coupling of mixtures of alkyl bromides of different molar compositions, synthesized by alkane bromination. To test this hypothesis, the non-selective radical monobromination of three linear alkanes 1a-c was performed in an efficient and scalable manner using $\mathrm{Br}_{2} / \mathrm{MnO}_{2}$ as a convenient system, ${ }^{[19]}$ and the corresponding mixtures of secondary alkyl bromides were employed in the migrative cross-coupling with various electrophiles (Scheme 3b). Excellent results were obtained with $n$-pentane 1a, $n$-hexane $\mathbf{1 b}$ and even dodecane 1c, which furnished the five linear products $\mathbf{3 c}, \mathbf{3} \mathbf{a h}$ and $\mathbf{3 a i}-\mathbf{a k}$, respectively, with good to high $1 / b$ selectivities depending on the employed aryl triflate. Gratifyingly, the scale of the bromination/regioconvergent coupling giving rise to product 3ak could be increased tenfold without significant decrease of the efficiency $(61 \%$ yield on 10 mmol vs. $67 \%$ on $1 \mathrm{mmol}$ of aryl triflate).

In conclusion, we have developed a cross-coupling method based on ligand-induced Pd migration, which allows to obtain functionalized alkanes from easily accessible branched alkyl bromides with good to high selectivity for the linear product. Highly practical Barbier-type conditions have been devised to in situ generate and cross-couple the intermediate organozinc species. Various positional isomers of the same alkyl bromide gave rise to the linear cross-coupling product with a comparable selectivity. This regioconvergent coupling was coupled to a non-selective radical monobromination step, thereby enabling the synthesis of terminal arylated products in only two steps from linear alkanes. The current two-step $\mathrm{C}-\mathrm{H}$ functionalization method is cost-effective, chemoselective, and operates under mild conditions and at reasonable catalyst loading.

Received: ((will be filled in by the editorial staff)) Published online on ((will be filled in by the editorial staff))

\section{Acknowledgments}

This work was financially supported by Agence National de la Recherche (ANR-14-CE06-0001), the China Scholarship Council, the Swiss National Science Foundation (200021_165987) and the University of Basel. We thank Dr. Mouâd Alami, Dr. Grégory Danoun, Prof. Dr. Andreas Pfaltz and Prof. Dr. Thomas Ward for helpful suggestions and comments, and Dr. Daniel Häussinger for assistance with NMR experiments.

Keywords: $\mathrm{C}-\mathrm{C}$ coupling $\cdot$ palladium $\cdot$ remote functionalization

[1] a) R. Jazzar, J. Hitce, A. Renaudat, J. Sofack-Kreutzer, O. Baudoin, Chem.-Eur. J. 2010, 16, 2654-2672; b) J. F. Hartwig, J. Am. Chem. Soc. 2016, 138, 2-24.

[2] a) A. R. Dick, M. S. Sanford, Tetrahedron 2006, 62, 24392463; b) T. Newhouse, P. S. Baran, Angew. Chem. Int. Ed. 2011, 50, 3362-3374

[3] a) I. A. I. Mkhalid, J. H. Barnard, T. B. Marder, J. M. Murphy, J. F. Hartwig, Chem. Rev. 2010, 110, 890-931; b) J. F. Hartwig, Acc. Chem. Res. 2012, 45, 864-873.

[4] For a two-step alkane dehydrogenation/ isomerization/silylation or borylation strategy: X. Jia, Z. Huang, Nature Chem. 2016, 8, 157-161. 
[5] J. D. Lawrence, M. Takahashi, C. Bae, J. F. Hartwig, J. Am. Chem Soc. 2004, 126, 15334-15335.

[6] a) A. Krasovskiy, V. Malakhov, A. Gavryushin, P. Knochel, Angew. Chem. Int. Ed. 2006, 45, 6040-6044; b) T. D. Blümke, F. M. Piller, P. Knochel, Chem. Commun. 2010, 46, 4082-4084.

[7] For recent Pd-catalyzed long-range cross-couplings and alkene isomerizations proceeding via a similar mechanism: a) B. J. Stokes, S. M. Opra, M. S. Sigman, J. Am. Chem. Soc. 2012, 134, 11408-11411; b) E. W. Werner, T.-S. Mei, A. J. Burckle, M. S. Sigman, Science 2012, 338, 1455-1458; c) T.-S. Mei, E. W. Werner, A. J. Burckle, M S. Sigman, J. Am. Chem. Soc. 2013, 135, 6830-6833; d) T.-S. Mei, H. H. Patel, M. S. Sigman, Nature 2014, 508, 340-344; e) B. J. Stokes, A. J. Bischoff, M. S. Sigman, Chem. Sci. 2014, 5, 2336-2339; f) E. Larionov, L. Lin, L. Guénée, C. Mazet, J. Am. Chem. Soc. 2014, 136, 16882-16894; g) H. H. Patel, M. S. Sigman, J. Am. Chem. Soc. 2015, 126, 3462-3465; h) C. Zhang, C. B. Santiago, L. Kou, M. S. Sigman, J. Am. Chem. Soc. 2015, 137, 7290-7293; i) L. Lin, C. Romano, C. Mazet, J. Am. Chem. Soc. 2016, 138, 10344-10350.

[8] For reviews on remote functionalization: a) I. Franzoni, C. Mazet, Org Biomol. Chem. 2014, 12, 233-241; b) A. Vasseur, J. Bruffaerts, I. Marek, I. Nature Chem. 2016, 8, 209-219.

[9] a) A. Renaudat, L. Jean-Gérard, R. Jazzar, C. E. Kefalidis, E. Clot, O. Baudoin, Angew. Chem. Int. Ed. 2010, 49, 7261-7265; b) P. Larini, C. E. Kefalidis, R. Jazzar, A. Renaudat, E. Clot, O. Baudoin, Chem. Eur. J. 2012, 18, 1932-1944; c) S. Aspin, A.-S. Goutierre, P. Larini, R.
Jazzar, O. Baudoin, Angew. Chem. Int. Ed. 2012, 51, 10808-10811; d) S. Aspin, L. López-Suárez, P. Larini, A.-S. Goutierre, R. Jazzar, O. Baudoin, Org. Lett. 2013, 15, 5056-5059.

[10] a) A. Millet, P. Larini, E. Clot, O. Baudoin, Chem. Sci. 2013, 4, 22412247 ; b) A. Millet, D. Dailler, P. Larini, O. Baudoin, Angew. Chem. Int. Ed. 2014, 53, 2678-2682.

[11] A. A. Fokin, P. R. Schreiner, Adv. Synth. Catal. 2003, 345, 1035-1052.

[12] a) A. Krasovskiy, C. Duplais, B. H. Lipshutz, J. Am. Chem. Soc. 2009 131, 15592-15593; b) A. Krasovskiy, C. Duplais, B. H. Lipshutz, Org. Lett. 2010, 12, 4742-4744.

[13] C. Duplais, A. Krasovskiy, A. Wattenberg, B. H. Lipshutz, Chem. Commun. 2010, 46, 562-564.

[14] V. R. Bhonde, B. T. O'Neill, S. L. Buchwald, Angew. Chem. Int. Ed. 2016, 55, 1849-1853.

[15] J. H. Lee, Y. Kishi, J. Am. Chem. Soc. 2016, 138, 7178-7186.

[16] For a Ni/Pd/Zn multimetallic system: L. K. G. Ackerman, M. M. Lovell, D. J. Weix, Nature 2015, 524, 454-457.

[17] a) C. Han, S. L. Buchwald, J. Am. Chem. Soc. 2009, 131, 7532-7533; b) Y. Yang, K. Niedermann, C. Han, S. L. Buchwald, Org. Lett. 2014, 16, 4638-4641.

[18] See for instance: C. Sämann, V. Dhayalan, P. R. Schreiner, P. Knochel, Org. Lett. 2014, 16, 2418-2421.

[19] X. Jiang, M. Shen, Y. Tang, C. Li, Tetrahedron Lett. 2005, 46, 487489. 


\section{C-C Coupling}

S. Dupuy, K.-F. Zhang, A.-S. Goutierre

O. Baudoin* Page - Page

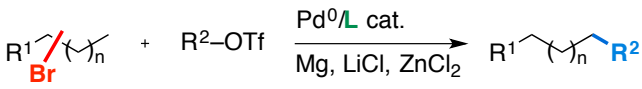

$$
\begin{aligned}
& \text { isolated or mixtures Barbier major linear } \\
& \text { of } 2^{\text {ary }} \text { alkyl bromides conditions product }
\end{aligned}
$$

Terminal-selective functionalization of alkyl chains by regioconvergent crosscoupling

Remote control: both isolated and mixtures of secondary alkyl bromides undergo regioconvergent Barbier-Negishi coupling with aryl triflates in the presence of a suitable phosphine ligand, giving rise to linear products with good to high linear/branched selectivities. 